\title{
New Stabiliser Systems for Highly Reliable Processes
}

\section{New stabiliser systems can increase the reliability and cost-effectiveness of parts cleaning processes. Adding the correct dose of stabiliser to the cleaning agent can extend the service life of the machine and the solvent.}

In many industries, parts cleaning is a quality-critical phase of production, which requires extensive expertise and a product and service concept that is coordinated with the entire manufacturing process. Safechem's Dowclene 16 range of distillable solvents consists of modified alcohols with a wide variety of applications. The company's newly developed stabiliser system (Maxistab S series) has extended the options for these cleaning products even further.

In addition to the contamination removed following machining processes, such as oils, greases and emulsions, other substances can be transferred into the cleaning machine which will have a negative effect on the process. As is the case with all distillable cleaning agents, there is the risk that the acid content of the medium will increase and that corrosion will occur on the parts and the machine. Stabilisers can now be used to counteract this process.

The newly developed stabiliser systems allow for non-contact stabilisation. The product is added to the steam generator and takes effect in the areas where potentially harmful substances could develop. As the stabiliser remains in the distillation residue and counteracts the development of acids and sulphur there, it does not come into contact with critical components of the cleaning machine. The consistent use of the stabiliser can reliably prevent problems with organic acids or sulphur compounds from occurring

\author{
The new stabiliser system allows cleaning \\ agents to be stabilised if they contain weak \\ organic acids and sulphur compounds or if they \\ are used to remove chlorinated oils.
}

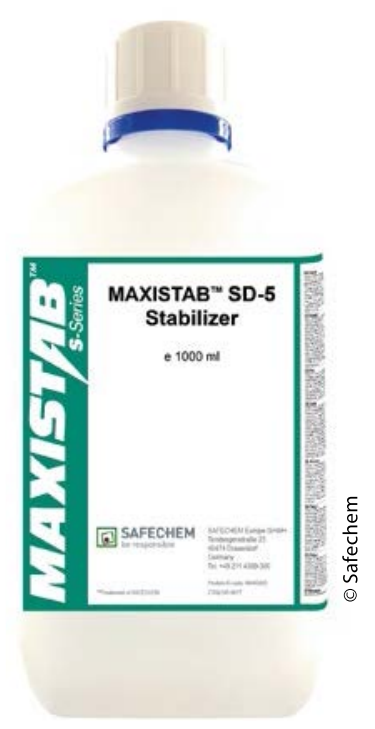

and can therefore help to extend the service life of delicate components, such as copper cooling units, seals, shut-off slides and valves. The service life of the solvent can also be increased. This means that the costs and downtimes resulting from maintenance, repairs and the replacement of the cleaning bath can be reduced.

Sulphurised oils often cause an unpleasant odour of bad eggs. This can be prevented by the Maxistab SD- 5 stabiliser. It also stops non-ferrous metals from becoming tarnished and discoloured when sulphurised oils are used.

\section{Reliable removal of chlorinated oils}

Chlorinated oils that are brought into the cleaning machine can cause hydrochloric acids and chlorides to form during the distillation process. This may lead to corrosion on the parts and the cleaning machine. It can also acidify the cleaning agent, which reduces its service life. In cases of this kind, the choice of the correct cleaning medium and of carefully coordinated stabilisers can significantly improve the quality, stability and cost-effectiveness of the cleaning process, which in turn can result in reproducible cleaning results.

Safechem's innovative two-component stabilising process, which consists of Maxistab SD-7 and Maxistab SV-9, makes it possible to remove high-grade chlorinated oils using the Dowclene 16 range of products. Maxistab SD-7 is added to the distillation unit and remains there. Maxistab SV-9 is volatile and performs its function throughout the entire cleaning system.

The correct dose of the stabiliser system ensures that the alkalinity of the cleaning medium remains reliably stable and contributes to a long service life for the machine and the solvent. A new Maxicheck test kit contains the necessary equipment for regular monitoring of the process. //

\section{Contact}

\section{Safechem Europe GmbH}

Düsseldorf, Germany

Tel. +492114389300

service@safechem.com

www.safechem.com 